Research Paper

\title{
UCHLI Is a Putative Tumor Suppressor in Ovarian Cancer Cells and Contributes to Cisplatin Resistance
}

\author{
Chengmeng Jin ${ }^{1,2}$, Wei Yu ${ }^{3}$, Xiaoyan Lou ${ }^{1}$, Fan Zhou ${ }^{1}$, Xu Han³ ${ }^{3}$ Na Zhao1, Biaoyang Lin 1,2,5, $\bowtie$ \\ 1. Systems Biology Division and Propriumbio Research Center, Zhejiang-California International Nanosystems Institute (ZCNI), Zhejiang \\ University, Hangzhou, Zhejiang Prov., China; \\ 2. Collaborative Innovation Center for Diagnosis and Treatment of Infectious Diseases, Zhejiang University, Hangzhou, China, \\ 3. Department of Biology, Technische Universität Darmstadt, Darmstadt Germany; \\ 4. Life Sciences Institute, Zhejiang University, Hangzhou, Zhejiang Prov., China; \\ 5. Swedish Neuroscience Institute, Swedish Medical Center, Seattle, WA 98122, USA; \\ 6. Department of Urology, University of Washington, Seattle, WA 98195, USA.
}

$\triangle$ Corresponding author: biaoylin@gmail.com

(C) Ivyspring International Publisher. This is an open-access article distributed under the terms of the Creative Commons License (http:/ / creativecommons.org/ licenses/by-nc-nd/3.0/). Reproduction is permitted for personal, noncommercial use, provided that the article is in whole, unmodified, and properly cited.

Received: 2013.05.07; Accepted: 2013.07.16; Published: 2013.09.27

\begin{abstract}
Ubiquitin carboxyl terminal hydrolase I ( $\mathrm{UCHLI}$ ) catalyzes the hydrolysis of $\mathrm{COOH}$-terminal ubiquityl esters and amides. It has been reported as either an oncogene or a tumor suppressor in cancers. However, UCHLI's role in ovarian cancer is still unclear. Therefore, we conducted an analysis to understand the role of UCHLI in ovarian cancer. Firstly, we detected UCHLI promoter methylation status in 7 ovarian cancer cell lines. 4 of them with UCHLI silencing showed heavy promoter methylation while the other 3 with relative high $U \mathrm{CHLI}$ expression showed little promoter methylation. Then we reduced UCHLI expression in ovarian cancer cell line A2780 and IGROVI and found that inhibition of UCHLI promoted cell proliferation by increasing cells in $\mathrm{S}$ phases of cell cycle. Knockdown of UCHLI also reduced cell apoptosis and contributed to cisplatin resistance. Furthermore, the expression level of UCHLI in several ovarian cancer cell lines correlated negatively with their cisplatin resistance levels. Microarray data revealed that UCHLI related genes are enriched in apoptosis and cell death gene ontology (GO) terms. Several apoptosis related genes were increased after UCHLI knockdown, including apoptosis regulator $B C L 2, B C L I I A, A E N$ and XIAP. Furthermore, we identified up-regulation of $\mathrm{Bcl}-2$ and $P A K T$ as well as down-regulation of Bax in UCHLI knockdown cells, while no significant alteration of $p 53$ and AKTI was found. This study provides a new and promising strategy to overcome cisplatin resistance in ovarian cancer via UCHLI mediated pathways.
\end{abstract}

Key words: Ubiquitin carboxyl terminal hydrolase 1, tumor suppressor

\section{Introduction}

Ovarian cancer is a serious health problem for women all over the world. Due to non-obvious early symptoms, about $70 \%$ of all cases are diagnosed at advanced stages ${ }^{1}$. In the USA, estimated new cases of ovarian cancer in 2012 account for only 3\% of all kinds of female cancer patients and it's estimated death rate doubles, reaching $6 \% 2$, which is higher than other gynecological malignancies.

UCHL1, short for ubiquitin carboxyl-terminal hydrolase L1, is a member of ubiquitin carboxy ter- minal hydrolase $(\mathrm{UCH})$ family that catalyzes the hydrolysis of $\mathrm{COOH}$-terminal ubiquityl esters and amides. UCHL1 was initially found to be expressed specifically in testis/ovary and brain ${ }^{3,4}$. UCHL1 mutations are related to neurodegenerative diseases such as Parkinson's diseases (PD) and Alzheimer disease (AD) ${ }^{5}$. UCHL1 has been reported as either an oncogene or a tumor suppressor gene. UCHL1 has been reported to be up-regulated in a variety of cancers. For example, over expression UCHL1 in lymphoma 
strongly accelerated lymphomagenesis by boosting signaling through the AKT pathway via down regulation of the antagonistic phosphatase PHLPP1 ${ }^{6}$. In non-small lung cancer cell line H157, UCHL1 promotes invasion by upstream activation of Akt 7 . In colorectal cancer, UCHL1 acts as an oncogene via activation of the beta-catenin/TCF pathway through its deubiquitinating activity ${ }^{8}$. In contrary, promoter hypermethylation that leads to silencing or low expression of UCHL1 were also found in several types of cancers, including nasopharyngeal carcinoma ${ }^{9}$, melanoma 10, hepatocellular carcinoma 11, esophageal squamous cell carcinoma 12, renal cancer 13, gastric cancer ${ }^{14}$ and prostate cancer ${ }^{15}$.

Cisplatin is a common reagent used in chemotherapy for ovarian cancers with an initial response rate of about $70 \%$, yet recurrence is common as patients often develop resistance to cisplatin therapy ${ }^{16}$. Proteins modulating cisplatin responses are of great importance to overcome cisplatin resistance. UCHL1 was a protein identified as differentially expressed proteins between the cisplatin-sensitive (A2780S) and cisplatin-resistant (A2780-CP) ovarian cancer cell lines ${ }^{17}$. However, the mechanism of how UCHL1 modulating cisplatin resistance has not been established. Here we describe our study in helping understanding the role of UCHL1 in chemotherapy response of ovarian cancers. We first showed that silencing of UCHL1 in ovarian cancer cell lines were caused by its promoter methylation and treatment of demethylation reagent could restore its expression. Furthermore, UCHL1 knockdown in ovarian cancer cell lines A2780 and IGROV1 promoted cell growth while causing reduction of apoptosis. Microarray analysis demonstrated that UCHL1 knockdown induced alterations in apoptosis and cell death. UCHL1 knockdown ovarian cancer cells (A2780-shUCHL1 and IGROV1-shUCHL1) became more resistant to cisplatin than their corresponding controls. Moreover, we found that UCHL1 expression level was negatively correlated with cisplatin resistance in ovarian cancer cells. We then detected up-regulation of Bcl-2 and down-regulation of Bax in UCHL1 knockdown cells, however, no significant alteration of p53 was detected. Also we detected up-regulation of phospho AKT. These results suggest that UCHL1 is mediator of apoptosis and targeting UCHL1 might be of great potential to overcome cisplatin resistance in ovarian cancer.

\section{Materials and Methods}

\section{I Cell lines and cell culture}

Ovarian cancer cell lines A2780, A2780CP, SKOV3 and IGROV1 were kind gifts from Dr. Stephen
Collins from UC San Diego (CA, USA). ES-2, OVCAR3 and CAOV3 were purchased from Cellbank, Chinese Academy of Sciences, Shanghai, China. ES-2 and SKOV3 were cultured in McCoy's 5A medium (Invitrogen, Carlsbad, CA) with 10\% FBS, while other cell lines were cultured in RPMI 1640 (Invitrogen) medium supplemented with $10 \% \mathrm{FBS}$ at $37^{\circ} \mathrm{C}$ and $5 \%$ $\mathrm{CO}_{2}$.

\subsection{Lentiviral vector and infection}

UCHL1 was knocked down by pLKO.1-puro lentiviral Vector cloned with UCHL1-target shRNA (CCGGGTGTGAGCTTCAGATGGTGAACTCGAGT TCACCATCTGAAGCTCACACTTTTT), purchased from Sigma-Aldrich. A Non-Mammalian shRNA (SHC002V) was used as a negative control. The lentivirus or the control was added to A2780 and IGROV1 cells with $8 \mu \mathrm{g} / \mathrm{ml}$ (final concentration) hexadimethrine bromide (H9268, Sigma-Aldrich) for infection. $500 \mathrm{ng} / \mathrm{ml}$ (final concentration) puromycin was added to select positive clones. Stable transfectants were isolated after 3 weeks selection.

\subsection{MTT assay and Cytotoxicity assay}

Growth was measured by MTT assay. Briefly, 3,000 cells were collected and seeded to each well of 96-well plate. After 1, 3, 5 days, MTT was added to cells (final concentration was $0.5 \mathrm{mg} / \mathrm{ml}$ ). After 4 hours incubation, medium was replaced by $100 \mathrm{ul}$ DMSO and OD value at $490 \mathrm{~nm}$ was measured.

To measure cisplatin sensitivity, 8,000 cells were seeded to each well in 5 replicates of 96-well plate. Cells were treated with a series dose of cisplatin from $0 \mu \mathrm{g} / \mathrm{ml}$ to $16 \mu \mathrm{g} / \mathrm{ml}$ and 48 hours later MTT assay was performed to measure cell viability. To induce apoptosis to measure active caspase-3 protein level, $20 \mu \mathrm{g} / \mathrm{ml}$ cisplatin (final concentration) was added and 16 hours later cells were harvested to perform western blot analysis.

\subsection{RNA isolation and Real-time PCR}

Total RNAs were extracted from cells using Trizol reagent (Invitrogen) according to the manufacturer's instructions. $2 \mu \mathrm{g}$ RNA was reverse transcribed in a final volume of $25 \mu 1$ using M-MLV reverse transcriptase (Promega, USA). Real-time PCR were performed using SYBR-Green PCR kit (Takara, Japan) in Bio-RAD CFX96 Real-Time System. All measurements were performed in triplicate following the cycle scheme: $95^{\circ} \mathrm{C}$ for $2 \mathrm{~min}, 40$ cycles of $95^{\circ} \mathrm{C}$ for $15 \mathrm{~s}, 58{ }^{\circ} \mathrm{C}$ for $30 \mathrm{~s}$ and $72^{\circ} \mathrm{C}$ for $15 \mathrm{~s}$. Primers for real-time PCR were designed as follows: UCHL1, forward, 5'- CCGAGATGCTGAACAAAG-3', and reverse, 5'-CAGAGACTCCTCTTCCAG-3'; BCL2, forward, 5'-ATAACGGAGGCTGGGATG-3', reverse, 5'-GCATGTTGACTTCACTTGTG-3'; GAPDH, for- 
ward, 5'-GGAGATTGTTGCCATCAACG-3', and reverse, 5' ${ }^{\prime}$-TTGGTGGTGCAGGATGCATT-3' .

\subsection{5-Aza-2'-deoxycytidine treatment}

A2780CP cell line was treated with $10 \mu \mathrm{M}$ 5-Aza-dC (Sigma-Aldrich) for 5 days. Medium was replaced with fresh 5-Aza-dC every 24 hours.

\subsection{DNA extraction, methylation specific PCR and bisulfite-modified DNA sequencing}

Genomic DNA was extracted from cultured cells using DNeasy Blood \& tissue Kit (Qiagen, Germany). Then genomic DNA was quantified and $250 \mathrm{ng}$ of DNA was bisulfite-treated with EZ DNA Methylation-GoldTM Kit (Zymo Research, CA, USA) according to the manufacturer's protocol. Methylation specific PCR (MSPCR) for UCHL1 was performed using Zymo Taq $^{\mathrm{TM}}$ DNA polymerase (Zymo Research) with primers as follows: $\mathrm{M}$, forward, 5'-TTTATTTGGTCGCGATCGT TC-3', reverse, 5'-AAACTACATCTTCGCGAAACG-3'; U, forward, 5'-GTATTTA TTTGGTTGTGATTGTTT-3', reverse, 5'-CTTAAACTACATCTTCACAAAAC A-3'. The MSPCR was carried out following the polymerase's protocol with annealing temperature $57^{\circ} \mathrm{C}$.

For bisulfite-modified DNA sequencing, Zymo $\mathrm{Taq}^{\mathrm{TM}}$ DNA polymerase was used to amplify a fragment of the UCHL1 promoter CpG islands containing $34 \mathrm{CpG}$ sites with primers as follows: forward, 5'-GTTTGGTTTTGTTTTT GTTTTTTTT-3', reverse, 5'-ACTTAATCCCTACCAACAACC-3'. The PCR cycle scheme was the same as MSPCR. PCR products were then cloned into pMD® $18-\mathrm{T}$ Vector (Takara) and 6-8 colonies were randomly chosen for sequencing. Primers for both MSPCR and bisulfite PCR were designed by web tools MethPrimer (http://www.urogene.org/cgi-bin/methprimer/met hprimer.cgi) ${ }^{18}$.

\subsection{Flow cytometry analysis of cell cycle and apoptosis}

For cell cycle assay, cells were cultured in 12 -well plates to about $70 \%$ in reduced serum (1\%) for 24 hours to get synchronized. Then cells were trypsinized and harvested, and washed with cold PBS. Next, cells were fixed with $80 \%$ cold ethanol over night at $4{ }^{\circ} \mathrm{C}$. Fixed cells were centrifuged and resuspended in propidium iodide solution, and subjected to flow cytometry analysis after 30 minutes' incubation at $37^{\circ} \mathrm{C}$.

For apoptosis analysis, cells $(10,000 /$ well) were cultured in 6-well plates to about $70 \%$ confluence then were cultured for 24 hours in reduced serum $(1 \%)$. Then cells were collected and Annexin V-FITC/PI staining was performed according to standard pro- tocols. After staining, cells were analyzed using BD FACS Array ${ }^{\mathrm{TM}}$ (Becton-Dickinson, San Jose, CA) according to manufacturer's guidelines.

\subsection{Western blot}

Protein was extracted using RIPA $[50 \mathrm{mM}$ Tris(pH 7.4), $150 \mathrm{mM} \mathrm{NaCl}, 1 \%$ Triton $\mathrm{X}-100,1 \%$ sodium deoxycholate, $0.1 \%$ SDS, $2 \mathrm{mM}$ sodium pyrophosphate, $25 \mathrm{mM} \beta$-glycerophosphate, 1mM EDTA, $1 \mathrm{mM} \mathrm{Na} 3 \mathrm{VO} 4,0.5 \mu \mathrm{g} / \mathrm{ml}$ leupeptin] and concentration was measured with BCA Protein Assay Kit (23225, Thermo Scientific). Equal amounts of protein $(20 \mu \mathrm{g})$ were added to $12 \%$ SDS-PAGE gel for electrophoresis. After that, proteins were transferred to polyvinylidene fluoride (PVDF) membranes and blocked in 5\% non-fat milk in TBST for 1 hour. Antibodies used were UCHL1 (PAB3903, Abnova), Bax (Epitomics), BCL2 (Epitomics), GAPDH (Epitomics). GAPDH was used as internal control.

\subsection{Microarray and Gene Ontology analysis}

To identify genes regulated by UCHL1, we performed a microarray assay using Affymetrix U133 plus 2 genechip (Affymetrix, Santa Clara, CA, USA). After RMA normalization, data were exported to the GeneSpring program (Agilent, Inc.) to filter out lowly expressed and unreliably detected genes with probe intensity $<100$. After that, genes with fold change $<2$ was furthered eliminated. Finally, Gene Ontology analysis was performed on line using the GoMiner program ${ }^{19}$.

\section{I 0 Statistical analysis}

All data were presented with mean \pm SD. Cell survival curves were fitted to the data points with nonlinear regression analysis using GraphPad Prism 5.0. Two-tailed Student's t-test was used to compare mean values. $p<0.05$ was considered statistically significant.

\section{Results}

\section{I UCHLI expression was correlated with its promoter methylation and could be restored with 5-Aza-dC treatment}

UCHL1 was reported frequently methylated in various cancers, including ovarian cancer ${ }^{20}$. To validate its promoter methylation status, we performed MS-PCR in 7 ovarian cancer cell lines (ES-2, A2780, IGROV1, OVCAR3, SKOV3, A2780CP and CAOV3). We found that the methylation status of UCHL1 in the promoter region correlated negatively with UCHL1 expression: those cell lines with very low UCHL1 expression showed positive methylation while those with relatively high UCHL1 expression showed negative methylation (Fig.1A). We next performed bisul- 
fite genomic sequencing (BGS) of all 7 ovarian cancer cell lines (Fig. 1B). BGS data matched perfectly with MS-PCR results (Fig. 1C): The MS-PCR positive cell lines showed heavy promoter methylation while MS-PCR negative cell lines showed very weak or no promoter methylation (Fig.1B-C).

We then chose A2780CP, an isogenic cell line of A2780 that differ in cisplatin resistance, and treated A2780CP cells with demethylation reagent 5-Aza-dC for 5 days to confirm whether silencing of UCHL1 is caused by its promoter hypermethylation. After 5-Aza-dC treatment, A2780CP showed a weak band of unmethylation (Fig.1D). Furthermore, UCHL1 expression was significantly induced after 5-Aza-dC treatment by RT-PCR analysis (Fig.1E). These results suggest that hypermethylation of UCHL1 in promoter region is responsible for its transcriptional silencing.

\subsection{Reduced expression of UCHLI in A2780 and IGROVI reduced cell apoptosis but in- creases cell proliferation}

To study the function of UCHL1 in ovarian can-

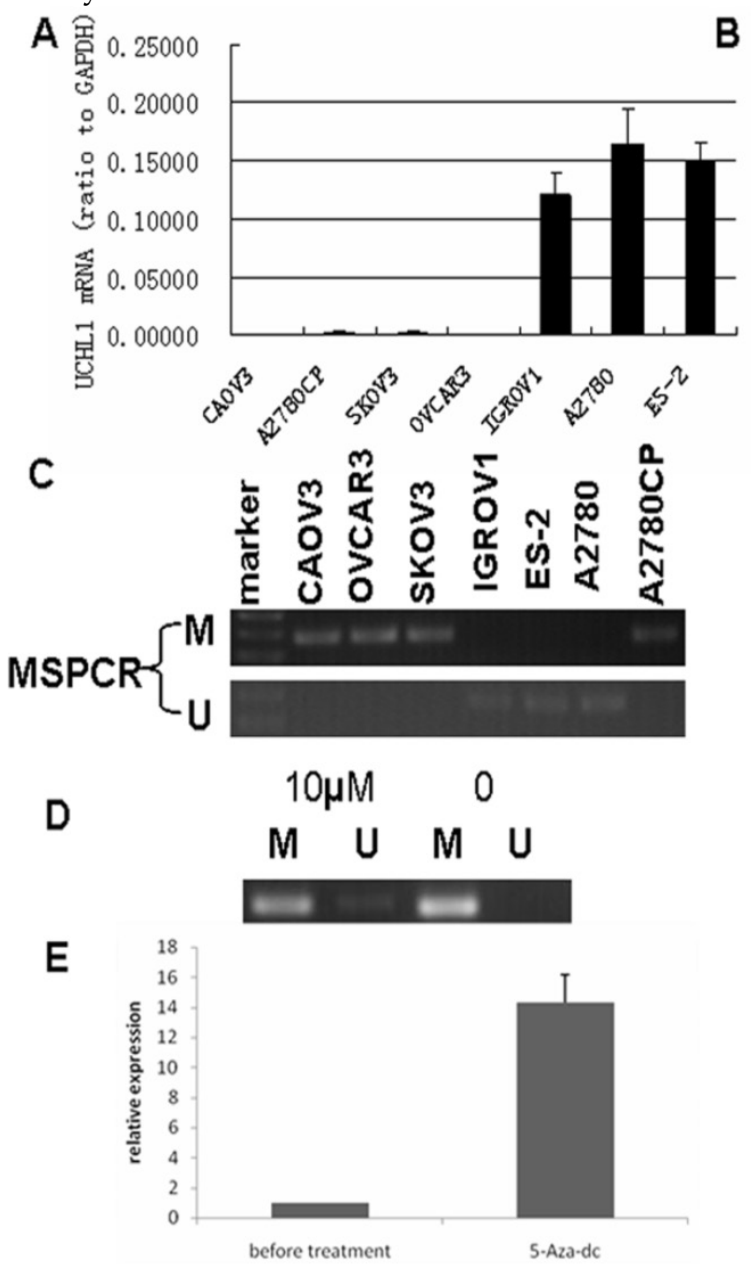

cer, two ovarian cancer cell lines, A2780 and IGROV1, which express relatively high level UCHL1, were chosen to perform UCHL1 knockdown. Lentivirus infection led to a high and stable knockdown-efficiency in both A2780 and IGROV1 cells. UCHL1 expression was significantly reduced at mRNA level as well as protein level (Fig. 2).

We performed a cell growth curve assay to determine the effect of UCHL1-knockdown in A2780 and IGROV1 cells. As shown in Fig. 3A, MTT absorbance at $490 \mathrm{~nm}$ of A2780-shUCHL1 and A2780-control differed significantly on the $5^{\text {th }}$ day, indicating that reduced expression of UCHL1 in A2780 cells increased cell proliferation. The same situation was detected between IGROV1-shUCHL1 and IGROV1-control. Furthermore, cell cycle analysis revealed up-regulation of $S$ phase both in A2780-shUCHL1 and IGROV1-shUCHL1 (Fig. 3B-C), indicating that UCHL1 knockdown promoted cell proliferation by progressing cell cycle.

\section{$B$}
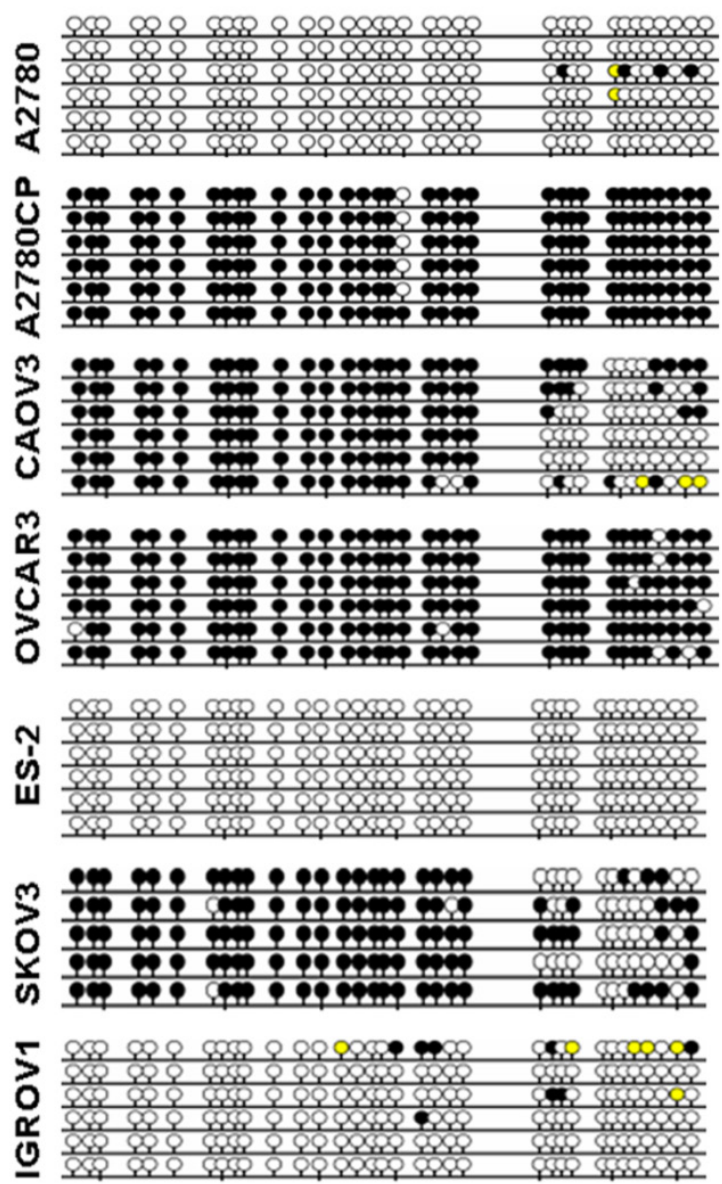

Fig I. Silencing of UCHLI was related to its promoter hypermethylation and UCHLI expression could be restored by 5-Aza-dc treatment. (A) UCHLI expression in seven ovarian cancer cell lines. (B) Bisulfite-modified DNA sequencing of UCHLI promoter in seven ovarian cancer cell lines. Open circles, unmethylated; closed circles, methylated; yellow, unknown methylation status. (C) Methylation status of UCHLI promoter in seven ovarian cancer cell lines. M, methylated; $U$, unmethylated. (D) Methylation status of UCHLI in A2780CP cells treated with/without $10 \mu M$ 5-Aza-dc. M, methylated; U, unmethylated. (E) UCHLI expression could be restored by 5-Aza-dc treatment in A2780CP cells. 
A

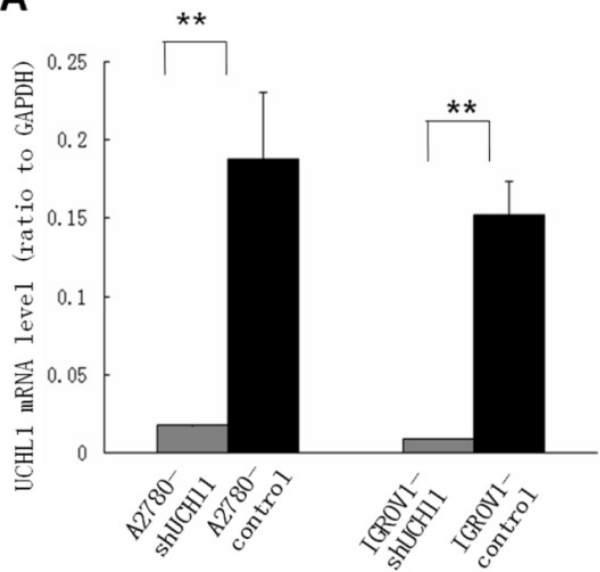

B

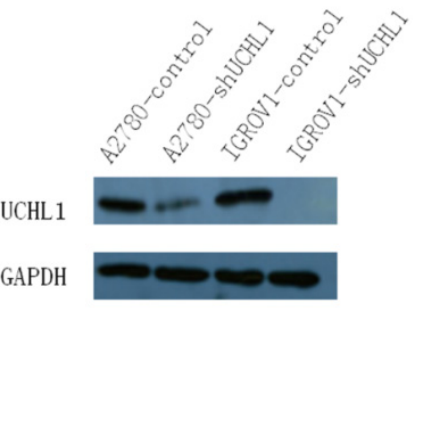

Fig 2. Suppression of UCHLI expression by shRNA. Lentivirus harboring shRNA against UCHLI or Non-Mammalian shRNA was used to infect UCHLI-positive A2780 and IGROVI cell lines. Expression of UCHLI was determined by quantitative real-time polymerase chain reaction (qRT-PCR) (A) and Western Blot (B). Both A2780-shUCHLI and IGROVI-shUCHLI showed a significant reduction of UCHLI expression. **, $p<0.0$ I.
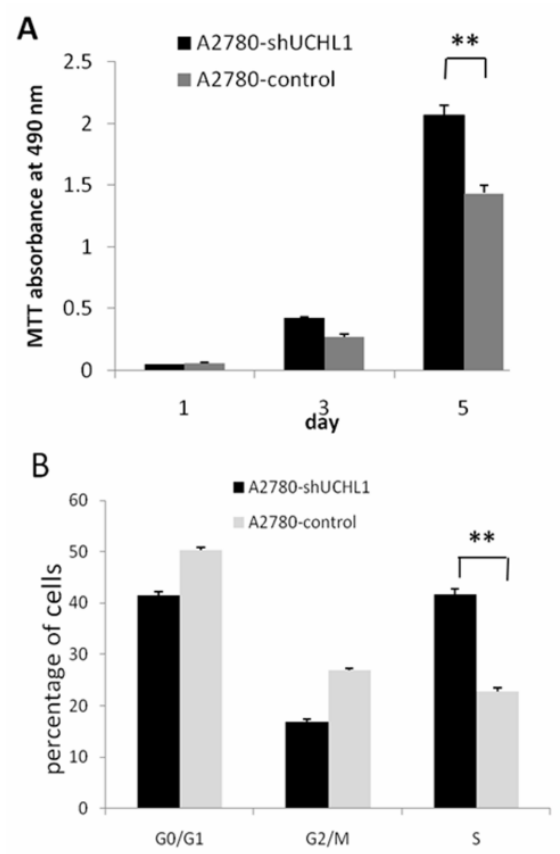
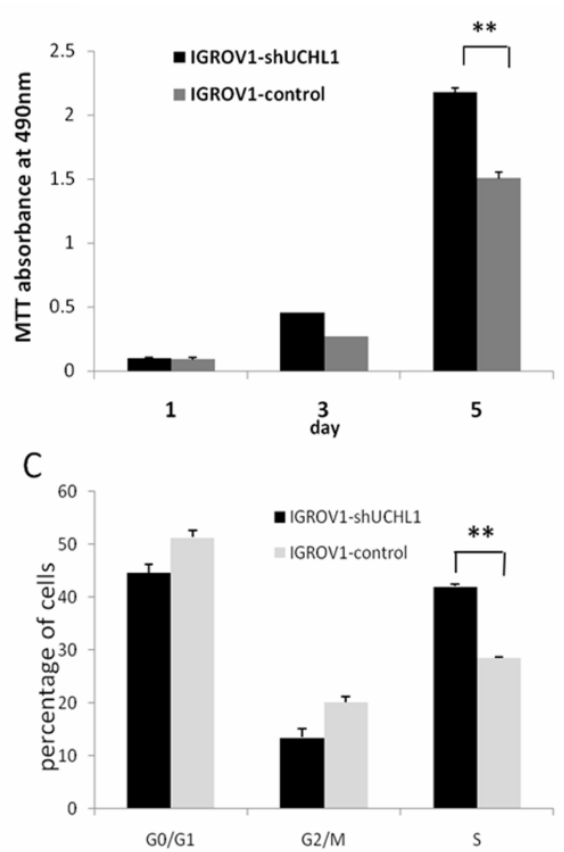

Fig 3. Effect of UCHLI knockdown on cell proliferation and cell cycle. (A) Cell proliferation ability was determined by MTT assay. Data was shown by mean of spectrometric absorbance $\pm S D$ of four independent experiments. (B) Cell cycle of A2780-shUCHLI and A2780-control was analyzed by flow cytometry. (C) Cell cycle of IGROVI-shUCHLI and IGROVI-control was analyzed by flow cytometry.

Then we assessed the effects of UCHL1 knockdown on apoptosis. We found that reduced UCHL1 expression resulted in reduction of apoptosis. In A2780-shUCHL1 cells, this effect was especially significant $(p<0.05)$ (Fig. 4A). IGROV1-shUCHL1 cells also showed a similar trend while the effect was not statistically significant (Fig. 4B).

\subsection{Reduced expression of UCHLI in A2780 and IGROVI induces cisplatin resistance}

Most anti-cancer reagents work by triggering apoptosis in tumor cells and the ability to induce apoptosis determines the therapeutic efficacy. Since suppression UCHL1 reduced apoptosis, we per- formed MTT assay to determine whether UCHL1 knockdown in ovarian cancer cells influences resistance to cisplatin, a common reagent used to treat ovarian cancer. As shown in Fig.4C, A2780-shUCHL1 and IGROV1-shUCHL1 showed relatively high cisplatin resistance compared to their controls, with $\mathrm{IC}_{50}$ increasing from $0.8 \mu \mathrm{g} / \mathrm{ml}$ to $2.4 \mu \mathrm{g} / \mathrm{ml}$ for A2780-control and A2780-shUCHL1, and from $1.2 \mu \mathrm{g} / \mathrm{ml}$ to $3.2 \mu \mathrm{g} / \mathrm{ml}$ for IGROV1-control and IGROV1-shUCHL1. Furthermore, we conducted western blot to determine cleaved caspase 3 expression after cisplatin treatment of these cells. As expected, there was a decrease in cleaved caspase 3 expression in UCHL1 knockdown cells (Fig.4D), indi- 
cating that reduced expression of UCHL1 in A2780 and IGROV1 made the cells become resistant to cisplatin-induced apoptosis.

We questioned whether UCHL1 expression level correlates with cisplatin resistance in ovarian cancer. We measured UCHL1 mRNA expression level and determined cisplatin resistance with MTT assay in a panel of ovarian cancer cell lines. SKOV3, OVCAR3 are derived from metastatic tumors of patients treated with chemotherapy and are resistant to cisplatin.
CAOV3 also exhibited cisplatin resistance although it's sensitive to other chemotherapy agent ${ }^{21}$. A2780, ES-2, and IGROV1, derived from untreated patients, are sensitive to cisplatin ${ }^{22,23}$. $\mathrm{A} 2780 \mathrm{CP}$, a parental cell line of A2780, is resistant to cisplatin ${ }^{24}$. We found that UCHL1 expression levels were negatively correlated with $\mathrm{IC}_{50}$ values from cisplatin MTT assay with a correlation coefficient of $-0.96(* *, p<0.001)$, which was statistically significant (Fig. 4E).

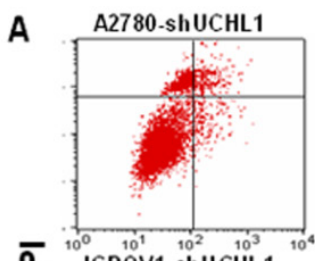

$\overline{\mathbf{L}}$

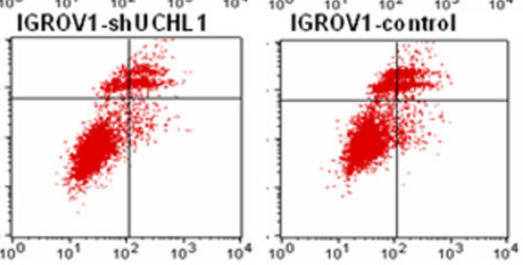

Annexin V

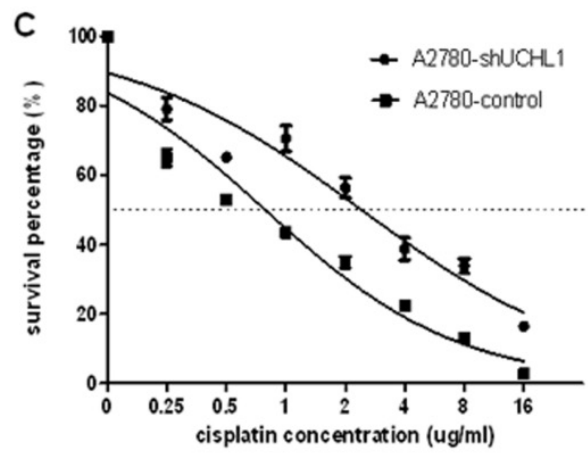

D

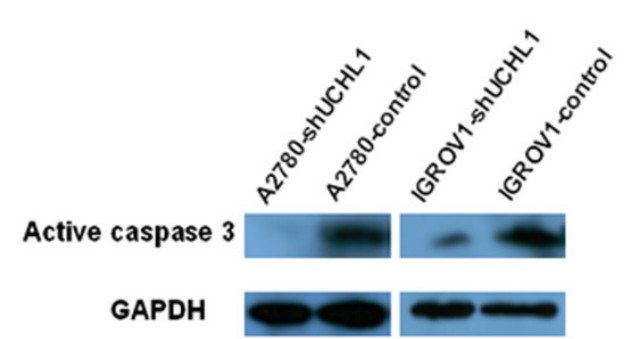

B
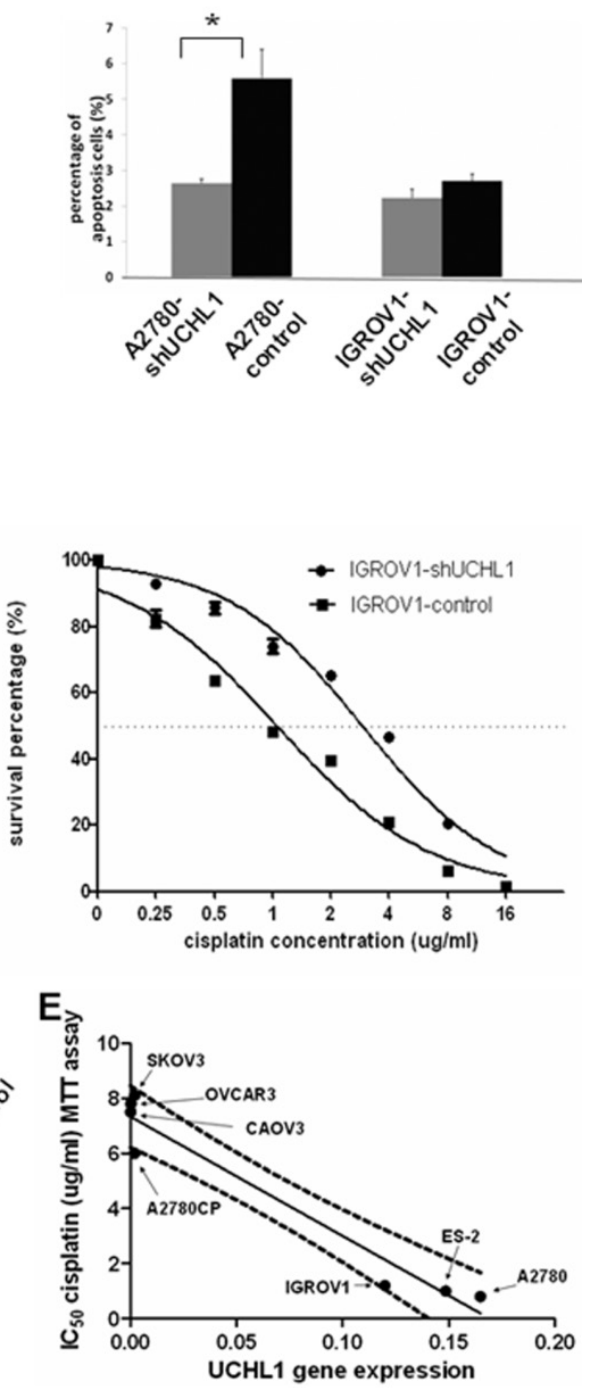

Fig 4. Knockdown of UCHLI reduced cell apoptosis and contributed to cisplatin resistance. (A) Cell apoptosis ratio was analyzed by flow cytometry. Shown were representative apoptosis figures of each cell line and data was summarized in (B). (C) UCHLI knockdown induced cisplatin resistance in ovarian cancer cell lines. A2780-shUCHLI, A2780-control, IGROVI-shUCHLI and IGROVI-control were treated with cisplatin in different doses from $0 \mu \mathrm{g} / \mathrm{ml}$ to $16 \mu \mathrm{g} / \mathrm{ml}$ for 48 hours. Then MTT assay was performed to measure the cell survival. Dotted line, $50 \%$ survival. (D) Total protein was extracted from the four cell lines which were treated with $20 \mu \mathrm{g} / \mathrm{ml}$ cisplatin for 16 hours and analyzed with active caspase 3 antibody, with GAPDH as internal control. (E) Expression of UCHLI in diverse ovarian cancer lines negatively correlates with cisplatin resistance. UCHLI mRNA expression and cisplatin resistance in a panel of 7 ovarian cancer cell lines were negatively correlated, with correlation coefficient $-0.96(p<0.001)$. Dotted line, $95 \%$ confidence interval. 


\subsection{Identification of biological process regu- lated by UCHLI suppression}

To explore the mechanisms of how reduced expression of UCHL1 promotes cell proliferation, reduces apoptosis and confers cisplatin resistance, we performed a microarray analysis to identify different genes regulated by UCHL1-knockdown. The array data was submitted to the GEO database (GSE43884) (http://www.ncbi.nlm.nih.gov/geo/query/acc.cgi?a $\mathrm{cC}=\mathrm{GSE} 43884)$. Comparing gene expression profile between A2780-shUCHL1 and A2780-control, we identified 1549 differentially expressed genes (724 down-regulated and 825 up-regulated) with fold change $>2$ (Supplementary Table 1). We noted up-regulation of several apoptosis related genes including apoptosis regulator BCL2 (B-cell CLL/lymphoma 2) (up 4.1 folds), BCL11A (B-cell CLL/lymphoma 11A) (up 29 folds), AEN (apoptosis enhancing nuclease) (up 2.0 folds) and XIAP (X-linked inhibitor of apoptosis) (up 2.6 folds).

We next focused on BCL2, an important regulator of apoptosis, for validation. First, we validated that BCL2 also was up-regulated at protein levels in knock-down cell lines A2780-shUCHL1 and
IGROV1-shUCH11 (Fig. 5A). Another member of BCL2 family Bax, which heterodimerizes with Bcl-2 to accelerate cell apoptosis 25 , was down-regulated (Fig. 5A). As p53 expression was reported to be altered in UCHL1 over-expression or knockdown cells 9, 11, 26, we also determined p53 level using western blot. However, we did not observe any significant alteration of its expression (Fig. 5A). Moreover, we detected higher pAKT expression in A2780-shUCHL1 and IGROV1-shUCHl1 while no significant alteration of AKT1 was detected, suggesting that the AKT pathway, rather than p53 signaling pathway might be involved in UCHL1 regulated apoptosis in these ovarian cancer cells.

Gene ontology analysis by GoMiner of these differentially expressed genes, with all gene list as total file, showed that 24 GO terms were found to be enriched, including positive regulation of apoptosis (GO:0043065), regulation of cell death (GO:0010942), negative regulation of apoptosis (GO:0043066), which were top three enriched GO terms (Table 1). This result was consistent with our observations, strongly indicating that UCHL1 is likely to be involved in the regulation of apoptosis.

Table I. Enriched GO terms in biological process for UCHLI regulated genes.

\begin{tabular}{|c|c|c|c|c|c|}
\hline GO Category & $\begin{array}{c}\text { Total } \\
\text { Genes }\end{array}$ & $\begin{array}{c}\text { Changed } \\
\text { Genes }\end{array}$ & Enrichment & LOG10 $(p)$ & $\begin{array}{c}\text { False } \\
\text { Discovery } \\
\text { Rate }\end{array}$ \\
\hline GO:0043065 positive regulation of apoptosis & 488 & 59 & 1.47 & -2.77 & 0.05 \\
\hline GO:0010942 positive regulation of cell death & 496 & 62 & 1.52 & -3.25 & 0.03 \\
\hline GO:0043066 negative regulation of apoptosis & 416 & 52 & 1.52 & -2.82 & 0.05 \\
\hline GO:0030097 hemopoiesis & 335 & 46 & 1.67 & -3.41 & 0.03 \\
\hline GO:0048534 hemopoietic or lymphoid organ development & 360 & 50 & 1.69 & -3.78 & 0.02 \\
\hline GO:0002520 immune system development & 388 & 51 & 1.60 & -3.26 & 0.03 \\
\hline GO:0007507 heart development & 245 & 35 & 1.74 & -3.04 & 0.05 \\
\hline GO:0060537 muscle tissue development & 180 & 28 & 1.89 & -3.11 & 0.04 \\
\hline GO:0014706 striated muscle tissue development & 171 & 28 & 1.99 & -3.48 & 0.03 \\
\hline GO:0001934 positive regulation of protein phosphorylation & 130 & 23 & 2.15 & -3.45 & 0.03 \\
\hline GO:0001932 regulation of protein phosphorylation & 235 & 37 & 1.91 & -4.03 & 0.01 \\
\hline GO:0051493 regulation of cytoskeleton organization & 153 & 28 & 2.22 & -4.34 & 0.01 \\
\hline GO:0045165 cell fate commitment & 144 & 24 & 2.03 & -3.18 & 0.04 \\
\hline GO:0045185 maintenance of protein location & 75 & 15 & 2.43 & -3.00 & 0.04 \\
\hline GO:0042594 response to starvation & 63 & 14 & 2.70 & -3.31 & 0.03 \\
\hline GO:0009267 cellular_response_to_starvation & 49 & 13 & 3.23 & -3.93 & 0.01 \\
\hline GO:0045580 regulation of $\mathrm{T}$ cell differentiation & 54 & 12 & 2.70 & -2.92 & 0.05 \\
\hline GO:0010332 response to gamma radiation & 29 & 10 & 4.19 & -4.19 & 0.01 \\
\hline GO:0048538 thymus development & 25 & 10 & 4.86 & -4.85 & 0.01 \\
\hline GO:0030856 regulation of epithelial cell differentiation & 33 & 10 & 3.68 & -3.66 & 0.03 \\
\hline GO:0043588 skin development & 33 & 9 & 3.32 & -2.98 & 0.05 \\
\hline GO:0048730 epidermis morphogenesis & 27 & 8 & 3.60 & -2.96 & 0.05 \\
\hline GO:0030035 microspike assembly & 31 & 9 & 3.53 & -3.19 & 0.04 \\
\hline GO:0031998 regulation of fatty acid beta-oxidation & 12 & 6 & 6.08 & -3.74 & 0.02 \\
\hline
\end{tabular}



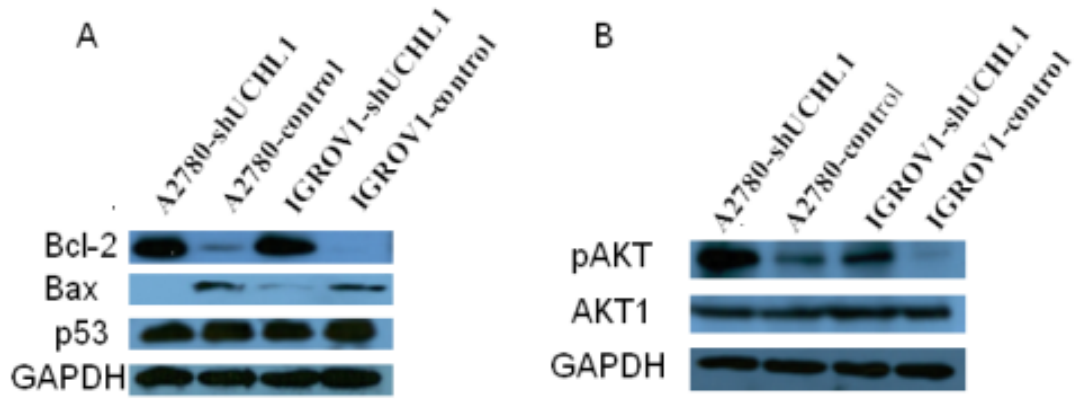

Fig 5. Detection of apoptosis related proteins by western blot. (A) Up-regulation of $\mathrm{Bcl}-2 / B a x$ but no significant alteration of $\mathrm{p} 53$ were detected in UCHLI knockdown cells. (B) Up-regulation of AKT pathway activity was found in UCHLI knockdown cells.

\section{Discussion}

In this study, we described, for the first time, how UCHL1 modulates cisplatin resistance in ovarian cancers. We showed that knockdown of UCHL1 in ovarian cancer cells promoted cell proliferation (Fig. $3 \mathrm{~A})$ by inducing $S$ phase cell cycle (Fig. 3B-C) and reduced apoptosis (Fig. 4A-B). Caspase 3, a frequently activated apoptosis protease and a typical hallmark of apoptosis 27 , is reduced upon UCHL1 knockdown (Fig. 4D). Our data is consistent with previous reports of UCHL1 in other types of cancers. UCHL1 over-expression has been reported to induce cell growth arrest in prostate cancer ${ }^{15}$ and breast cancer ${ }^{26 \text {, }}$ 28. In breast cancer, ectopic expression of UCHL1 is able to induce apoptosis by stabilizing p53. In nasopharyngeal carcinoma, UCHL1 was found to form a complex with p53/MDM2/ARF to promote p53 signaling ${ }^{9}$. In hepatocellular carcinoma, it was reported that UCHL1 serves as a tumor suppressor gene by directly interacting with p53 and stabilizing p53 ${ }^{11}$. Yet in our study, no significant alteration of p53 was found (Fig. 5A), suggesting UCHL1 mediated apoptosis in other ways rather than by regulating $\mathrm{p} 53$.

We initially observed that UCHL1 expression is anti-correlated with cisplatin resistant levels (as measured by $\mathrm{IC}_{50}$ ) (Fig. 4E). This is consistent with a UCHL1 expression at the GEO database showing that UCHL1 was reduced to no expression in the tissues from carboplatin resistant ovarian cancer patients (http://www.ncbi.nlm.nih.gov/geo/tools/profileGr aph.cgi?ID=GDS1381:36990_at) compared to the carboplatin sensitive tissues (Supplementary Fig. 1) ${ }^{29}$. Carboplatin, a parental platinum compound of cisplatin, shares similar biochemical characters as well as similar anti-cancer mechanisms with cisplatin ${ }^{30}$. The fact that cross resistance between carboplatin and cisplatin 31,32 ensure us making use of the GEO dataset.

We further observed that methylation of the UCHL1 promoter correlated negatively to the expres- sion of UCHL1 (Fig. 1A-C). Demethylation of A2780CP with Aza restored UCHL1 expression, confirming promoter methylation was responsible for its silencing (Fig. 1D-E). This is consistent with the previous observation by Okochi-Takada et al. who showed that UCHL1 was silenced in about half of the ovarian cancer cell lines that they tested and the expression was also correlated with promoter methylation status ${ }^{33}$. Moreover, we have extended this finding to further experimentation and showed that knockdown of UCHL1 increased cisplatin resistance in both A2780 and IGROV1 ovarian cancer cells (Fig. 4C). Our data demonstrated, for the first time, that UCHL1 expression directly regulated cisplatin resistance in ovarian cancers.

We also performed microarray expression profiling to understand the global picture of UCHL1's effects on ovarian cancer cells. GoMiner analysis showed that apoptosis and cell death related biological processes are enriched in UCHL1 regulated genes. In particular, we found that several apoptosis related genes including apoptosis regulator BCL2 (B-cell CLL/lymphoma 2), BCL11A (B-cell CLL/lymphoma 11A), AEN (apoptosis enhancing nuclease) and XIAP (X-linked inhibitor of apoptosis). AEN was recently identified as a direct target gene of p53 ${ }^{34}$. Bcl-2/Bax ratio is a determinant of cell fate and was considered to be a "rheostat" that regulates apoptosis ${ }^{35,36}$. We found up-regulated $\mathrm{Bcl}-2 / \mathrm{Bax}$ ratio in UCHL1-knockdown cells (Fig. 5A), however, no significant alteration of p53 was found (Fig. 5A).

AEN induces apoptosis by itself, and more importantly, it is also required for efficient DNA fragmentation in p53-dependent apoptosis ${ }^{34}$. The role of AEN in UCHL1 knockdown induced apoptosis remains to be studied. XIAP regulates AKT activity and caspase-3-dependent cleavage during cisplatin-induced apoptosis in human ovarian epithelial cancer cells ${ }^{37}$. Interestingly, in our study we found up-regulated AKT activity in UCHL1-knockdown 
ovarian cancer cells (Fig. 5B). Downregulation of XIAP inhibits proliferation, induces apoptosis, and reverses the cisplatin resistance of ovarian carcinoma 38. Here we demonstrated that UCHL1 regulated the expression of AEN and XIAP, suggesting that UCHL1 might be a key master regulator of cisplatin resistance in ovarian cancers. Further experimentation is warranted.

In summary, we present data showing that UCHL1 might be a tumor suppressor in ovarian cancers. Furthermore, UCHL1 expression level is negatively related to cisplatin resistance in ovarian cancer and knockdown of UCHL1 increased cisplatin resistance. GoMiner analysis showed that apoptosis and cell death related biological processes are enriched in UCHL1 regulated genes. Our study provides a foundation to develop promising strategy to overcome cisplatin resistant ovarian cancer via UCHL1 mediated pathways.

\section{Supplementary Material}

Supplementary Table 1

[http://www.jcancer.org/v04p0662s1.pdf]

Supplementary Figure 1

[http://www.jcancer.org/v04p0662s2.pdf]

\section{Acknowledgement}

This research was supported by grants from MOST, China (2012AA022705 and 2007DFC30360) and a grant from the Shaoxing Major Scientific and Technological Projects (2011A11013).

\section{Competing Interests}

Biaoyang Lin received research funding from Hangzhou Proprium Biotech Co. The other authors have declared that no competing interest exists.

\section{References}

[1] Naora H, Montell DJ. Ovarian cancer metastasis: integrating insights from disparate model organisms. Nat Rev Cancer. 2005; 5: 355-66.

[2] Siegel R, Naishadham D, Jemal A. Cancer statistics, 2012. CA: A Cancer Journal for Clinicians. 2012; 62: 10-29.

[3] Sekiguchi S, Yoshikawa Y, Tanaka S, et al. Immunohistochemical analysis of protein gene product 9.5, a ubiquitin carboxyl-terminal hydrolase, during placental and embryonic development in the mouse. Exp Anim Tokyo. 2003; 52: 365-9.

[4] Wilkinson KD, Lee K, Deshpande S, Duerksenhughes P, Boss JM, Pohl J. The Neuron-Specific Protein Pgp-9.5 Is a Ubiquitin Carboxyl-Terminal Hydrolase. Science. 1989; 246: 670-3.

[5] Setsuie R, Wada K. The functions of UCH-L1 and its relation to neurodegenerative diseases. Neurochem Int. 2007; 51: 105-11.

[6] Hussain S, Foreman O, Perkins SL, et al. The de-ubiquitinase UCH-L1 is an oncogene that drives the development of lymphoma in vivo by deregulating PHLPP1 and Akt signaling. Leukemia. 2010; 24: 1641-55.

[7] Kim HJ, Kim YM, Lim S, Nam YK, Jeong J, Lee KJ. Ubiquitin C-terminal hydrolase-L1 is a key regulator of tumor cell invasion and metastasis. Oncogene. 2008; 28: 117-27.

[8] Zhong J, Zhao M, Ma Y, et al. UCHL1 acts as a colorectal cancer oncogene via activation of the beta-catenin/TCF pathway through its deubiquitinating activity. Int J Mol Med. 2012; 30: 430-6.

[9] Li L, Tao Q, Jin H, et al. The Tumor Suppressor UCHL1 Forms a Complex with p53/MDM2/ARF to Promote p53 Signaling and Is Frequently Silenced in Nasopharyngeal Carcinoma. Clinical Cancer Research. 2010; 16: 2949-58.
[10] Bonazzi VF, Nancarrow DJ, Stark MS, et al. Cross-platform array screening identifies COL1A2, THBS1, TNFRSF10D and UCHL1 as genes frequently silenced by methylation in melanoma. PLoS One. 2011; 6: e26121.

[11] Yu J, Tao Q, Cheung KF, et al. Epigenetic identification of ubiquitin carboxyl-terminal hydrolase L1 as a functional tumor suppressor and biomarker for hepatocellular carcinoma and other digestive tumors. Hepatology. 2008; 48: 508-18.

[12] Mandelker DL, Yamashita K, Tokumaru Y, et al. PGP9.5 promoter methylation is an independent prognostic factor for esophageal squamous cell carcinoma. Cancer Res. 2005; 65: 4963-8.

[13] Seliger B, Handke D, Schabel E, Bukur J, Lichtenfels R, Dammann R. Epigenetic control of the ubiquitin carboxyl terminal hydrolase 1 in renal cell carcinoma. Journal of Translational Medicine. 2009; 7: 90.

[14] Yamashita K, Park HL, Kim MS, et al. PGP9.5 methylation in diffuse-type gastric cancer. Cancer Res. 2006; 66: 3921-7.

[15] Ummanni R, Jost E, Braig M, et al. Ubiquitin carboxyl-terminal hydrolase 1 (UCHL1) is a potential tumour suppressor in prostate cancer and is frequently silenced by promoter methylation. Mol Cancer. 2011; 10:129.

[16] Einhorn LH. Testicular cancer: An oncological success story. Clinical Cancer Research. 1997; 3: 2630-2.

[17] Gong F, Peng X, Zeng Z, Yu M, Zhao Y, Tong A. Proteomic analysis of cisplatin resistance in human ovarian cancer using 2-DE method. Mol Cell Biochem. 2011; 348: 141-7.

[18] Li LC, Dahiya R. MethPrimer: designing primers for methylation PCRs. Bioinformatics. 2002; 18: 1427-31.

[19] Zeeberg BR, Feng W, Wang G, et al. GoMiner: a resource for biological interpretation of genomic and proteomic data. Genome Biol. 2003; 4: R28.

[20] Okochi-Takada E, Nakazawa K, Wakabayashi M, et al. Silencing of the UCHL1 gene in human colorectal and ovarian cancers. Int J Cancer. 2006; 119: 1338-44.

[21] Petru E, Sevin BU, Perras J, et al. Comparative Chemosensitivity Profiles in 4 Human Ovarian-Carcinoma Cell-Lines Measuring Atp Bioluminescence. Gynecologic Oncology. 1990; 38: 155-60.

[22] Katano K, Kondo A, Safaei R, et al. Acquisition of resistance to cisplatin is accompanied by changes in the cellular pharmacology of copper. Cancer Research. 2002; 62: 6559-65.

[23] Wang Y, Niu XL, Qu Y, et al. Autocrine production of interleukin-6 confers cisplatin and paclitaxel resistance in ovarian cancer cells. Cancer Letters. 2010; 295: 110-23.

[24] Yu W, Jin CM, Lou XY, et al. Global Analysis of DNA Methylation by Methyl-Capture Sequencing Reveals Epigenetic Control of Cisplatin Resistance in Ovarian Cancer Cell. Plos One. 2011; 6.

[25] Oltvai ZN, Milliman CL, Korsmeyer SJ. Bcl-2 heterodimerizes in vivo with a conserved homolog, Bax, that accelerates programmed cell death. Cell. 1993; 74: 609-19.

[26] Xiang T, Li L, Yin X, et al. The Ubiquitin Peptidase UCHL1 Induces G0/G1 Cell Cycle Arrest and Apoptosis Through Stabilizing p53 and Is Frequently Silenced in Breast Cancer. PLoS One. 2012; 7: e29783.

[27] Porter AG, Janicke RU. Emerging roles of caspase-3 in apoptosis. Cell Death Differ. 1999; 6: 99-104.

[28] Liu XP, et al. Over-expression of ubiquitin carboxy terminal hydrolase-L1 induces apoptosis in breast cancer cells. International Journal of Oncology. 2008;33:1037-1045.

[29] Peters D, Freund J, Ochs RL. Genome-wide transcriptional analysis of carboplatin response in chemosensitive and chemoresistant ovarian cancer cells. Mol Cancer Ther. 2005; 4: 1605-16.

[30] Knox RJ, Friedlos F, Lydall DA, Roberts JJ. Mechanism of cytotoxicity of anticancer platinum drugs: evidence that cis-diamminedichloroplatinum(II) and cis-diammine-(1,1-cyclobutanedicarboxylato)platinum(II) differ only in the kinetics of their interaction with DNA. Cancer Res. 1986; 46: 1972-9.

[31] Gore ME, Fryatt I, Wiltshaw E, Dawson T, Robinson BA, Calvert AH. Cisplatin/carboplatin cross-resistance in ovarian cancer. Br J Cancer. 1989; 60: 767-9

[32] Boven E, van der Vijgh WJ, Nauta MM, Schluper HM, Pinedo HM. Comparative activity and distribution studies of five platinum analogues in nude mice bearing human ovarian carcinoma xenografts. Cancer Res. 1985; 45: 86-90.

[33] Okochi-Takada E, Nakazawa K, Wakabayashi M, et al. Silencing of theUCHL1 gene in human colorectal and ovarian cancers. International Journal of Cancer. 2006; 119: 1338-44

[34] Kawase $\mathrm{T}$, Ichikawa $\mathrm{H}$, Ohta $\mathrm{T}$, et al. p53 target gene AEN is a nuclear exonuclease required for p53-dependent apoptosis. Oncogene. 2008; 27: 3797-810.

[35] Korsmeyer SJ, Shutter JR, Veis DJ, Merry DE, Oltvai ZN. Bcl-2/Bax: a rheostat that regulates an anti-oxidant pathway and cell death. Semin Cancer Biol. 1993; 4: 327-32.

[36] Chresta CM, Masters JR, Hickman JA. Hypersensitivity of human testicular tumors to etoposide-induced apoptosis is associated with functional p53 and a high Bax:Bcl-2 ratio. Cancer Res. 1996; 56: 1834-41.

[37] Asselin E, Mills GB, Tsang BK. XIAP regulates Akt activity and caspase-3-dependent cleavage during cisplatin-induced apoptosis in human ovarian epithelial cancer cells. Cancer Res. 2001; 61: 1862-8.

[38] Ma JJ, Chen BL, Xin XY. XIAP gene downregulation by small interfering RNA inhibits proliferation, induces apoptosis, and reverses the cisplatin resistance of ovarian carcinoma. Eur J Obstet Gynecol Reprod Biol. 2009; 146: 222-6. 